\title{
Dasatinib-induced pulmonary arterial hypertension unresponsive to PDE-5 inhibition
}

\section{To the Editor:}

We read with interest the article "Src tyrosine kinase is crucial for potassium channel function in human pulmonary arteries" by NAGARAJ et al. [1]. The authors elegantly demonstrated that in physiological conditions, a low pulmonary vascular tone is maintained by Src family tyrosine kinase (SrcTK) and that inhibition of SrcTK will lead to vasoconstriction of pulmonary resistance arteries and thus to an increase in pulmonary artery pressure [1].

This finding fits well with the recent observation made in the French registry that dasatinib, a dual Src/Abl kinase inhibitor, can cause pulmonary arterial hypertension (PAH) in patients treated with this drug [2]. In some of those patients, PAH improved after withdrawal of the drug, supporting the notion that Srcmediated vasoconstriction might play a role in the development of dasatinib-induced pulmonary hypertension.

Here, we present another case of dasatinib-induced PAH. This case illustrates that dasatinib-induced PAH seems unresponsive to phosphodiesterase type 5 inhibition when dasatinib therapy is continued and is only reversed by withdrawing the drug. This case suggests that when inducing pulmonary vasoconstriction, dasatinib either acts downstream from the secondary messenger cGMP or acts via a cGMP-unrelated mechanism or independently from the classical pathobiological pathways leading to PAH [3].

A 57-year-old male was diagnosed with chronic myeloid leukaemia (CML) in 2002. Except for mild chronic obstructive pulmonary disease and hypertension, the patient had no other relevant diseases in his medical history. Imatinib treatment was initiated and because of side effects, switched to dasatinib in 2007. After 37 months of dasatinib treatment $\left(70 \mathrm{mg} \cdot\right.$ day $\left.^{-1}\right)$, the patient developed progressive dyspnoea and signs of ankle oedema. Although the ankle oedema was first classified as a side effect of dasatinib, progression of dyspnoea led to further evaluation.

After performance of an echocardiogram and an exercise test, suspicion of pulmonary hypertension rose. 2 months later, the diagnosis was confirmed by a right heart catheterisation. The mean pulmonary artery pressure (mPAP) was $55 \mathrm{mmHg}$ and the pulmonary vascular resistance was 721 dysnes $\cdot \mathrm{s}^{\cdot} \mathrm{cm}^{-5}$. Reversibility testing by NO was not performed. After exclusion of all possible causes, the diagnosis was made of pulmonary hypertension associated with a myeloproliferative disorder. The patient was referred to our institute for institution of treatment. At the time of admission, the patient was in New York Heart Association (NYHA) functional class 4, unable to perform exercise and showed signs of right heart failure. Although sildenafil treatment and diuretics were initiated the patient did not improve in NYHA class. After five months of unsatisfactory treatment, data emerged from the French registry that dasatinib can induce $\mathrm{PAH}$. Based on this information, Dasatinib was substituted for an alternative tyrosine kinase inhibitor nilotinib $800 \mathrm{mg} \cdot \mathrm{day}^{-1}$, leading to a quick and dramatic improvement in the patient's condition (NYHA 1). 3 months after discontinuation of dasatinib and sildenafil treatment, he had no symptoms and was able to resume his job. Repeated echocardiography showed that the pressure gradient over the tricuspid valve had decreased from 110 to $38 \mathrm{mmHg}$. The patient declined a second right heart catheterisation.

From this case, we can learn that PAH-specific therapy was not effective in dasatinib-associated PAH. This has not been previously reported, since the patients in the cases of MONTANI et al. [2] and DUMITRESCU et al. [4] immediately discontinued dasatinib when PAH was diagnosed.

The quick resolution of symptoms and improvement of pulmonary hypertension in our patient indicate that vasoconstriction rather than remodelling caused the PAH. Of interest is that sildenafil had no effect in our patient, whereas it is known that sildenafil can improve exercise capacity and reduce PAH in the case of hypoxic vasoconstriction [5]. Although no firm conclusions can be made from our observation, it strongly supports the findings of NAGARAJ et al. [1] that Src TK is a crucial factor for pulmonary vascular tone and mediates pulmonary vasodilation in a cGMP independent way. 
@ERSpublications

Src family tyrosine kinase is crucial in pulmonary vascular tone http://ow.ly/mGqEa

Joanne A. Groeneveldt ${ }^{1}$, Steven J. M. Gans ${ }^{2}$, Harm J. Bogaard ${ }^{1}$ and Anton Vonk-Noordegraaf ${ }^{1}$

${ }^{1}$ Dept of Pulmonary Diseases, VU University Medical Center, Amsterdam, and ${ }^{2}$ Dept of Pulmonary Diseases, Sint Jansdal, Harderwijk, The Netherlands.

Correspondence: J.A. Groeneveldt, VU University Medical Center, De Boelelaan 1117, 1081 HV Amsterdam, The Netherlands. E-mail: j.groeneveldt@vumc.nl

Received: Feb 262013 | Accepted after revision: Apr 282013

Conflict of interest: Disclosures can be found alongside the online version of this article at www.erj.ersjournals.com

\section{References}

Nagaraj C, Tang B, Bálint Z, et al. Src tyrosine kinase is crucial for potassium channel function in human pulmonary arteries. Eur Respir J 2013; 41: 85-95.

2 Montani D, Bergot E, Gunther S, et al. Pulmonary arterial hypertension in patients treated by dasatinib. Circulation 2012; 125: 2128-2137.

3 Voelkel NF, Gomez-Arroyo J, Abbate A, et al. Pathobiology of pulmonary arterial hypertension and right ventricular failure. Eur Respir J 2012; 40: 1555-1565.

4 Dumitrescu D, Seck C, ten Freyhaus H, et al. Fully reversible pulmonary arterial hypertension associated with dasatinib treatment for chronic myeloid leukaemia. Eur Respir J 2011; 38: 218-220.

Ghofrani HA, Reichenberger F, Kohstall MG, et al. Sildenafil increased exercise capacity during hypoxia at low altitudes and at Mount Everest base camp: a randomized, double-blind, placebo-controlled crossover trial. Ann Intern Med 2004; 141: 169-177.

\section{A case of resistance beyond extensively drug-resistant tuberculosis in Japan}

To the Editor:

We read with interest the recent article by MigLIORI et al. [1]. The study compares the treatment outcomes of extensively drug-resistant (XDR) tuberculosis (TB) patients with and without additional drug resistance, and provides informative data to develop reliable definitions for drug resistance beyond XDR-TB. The emergence and spread of multidrug resistant (MDR)-TB and XDR-TB poses a global threat. The first cases of extremely drug-resistant (XXDR)-TB were reported in Italy in 2007 [2], then subsequent cases of totally drug-resistant (TDR)-TB were reported in Iran in 2009 and India in 2012 [3, 4], although the definition of XXDR or TDR remains controversial $[5,6]$.

In Japan, the prevalence of MDR-TB and XDR-TB among TB patients surveyed was $1.9 \%$ and $0.5 \%$, respectively, according to a nationwide survey in 2002 [7]. According to an article by MURASE et al. [8] a high frequency $(71 \%)$ of XDR-TB strains are clustered, suggesting that the transmission of XDR-TB is occurring in communities in Japan. Additionally, of the $55 \mathrm{MDR} / \mathrm{XDR}-\mathrm{TB}$ cases, four were identified as TDR-TB, which were resistant to all 10 drugs tested [8], but little clinical information was provided on these cases. We report on a beyond-XDR-TB case in Japan that developed after 13 years of anti-TB treatment.

A male patient in his 50s with XDR-TB was admitted to our hospital and placed in a negatively-pressured isolation room in May 2011, because he had a positive, acid-fast bacilli (AFB) sputum. He had a previous history of hypertension, diabetes, alcoholism, and chronic pancreatitis. The patient was first diagnosed with pulmonary TB in January 1998 and initiated with a three-drug regimen of isoniazid, rifampicin, and ethambutol. However, this was changed to a four-drug regimen of isoniazid, pyrazinamide, ethionamide and levofloxacin in May 1998, because the initial drug-susceptibility testing (DST) showed poly-resistance that included isoniazid, rifampicin, and ethambutol. Pyrazinamide was stopped due to liver dysfunction. The patient had a history of receiving various treatments for $\mathrm{TB}$, and had been treated with first-line drugs (isoniazid, rifampicin, ethambutol, streptomycin, and/or pyrazinamide) and other drugs (cycloserine, ethionamide, levofloxacin, and/or sultamicillin) at several hospitals; however, repeated sputum samples remained positive. The patient may have amplified his resistance because of inadequate regimens. Surgical lobectomy was considered because the lesion was limited to the left lobe; however, the patient refused the 